\title{
On the existence of weak solutions for a class of singular reaction diffusion systems
}

\author{
Salim Mesbahi \\ LMFN Laboratory, Department of Mathematics, Faculty of Sciences, Ferhat Abbas University, Setif, \\ Algeria
}

\begin{abstract}
We study the existence of weak solutions for a parabolic reaction diffusion model applied in Quenching endowed with singular production terms by reaction. The singularity is due to a potential occurrence of quenching localized to the domain boundary. The techniques used are based on energy estimates to approach nonsingular problems and uniform control on the set where singularities are localizing.
\end{abstract}

Mathematics Subject Classification (2020). 35K57, 35K67, 35D30

Keywords. reaction diffusion systems, singular parabolic equations, weak solutions, quenching

\section{Introduction}

The applications of reaction diffusion systems are numerous, particularly in the modeling of real diffusion phenomena in biology, chemistry and engineering, see Levenspiel [19] and Murray [27,28]. A very interesting case is that of quenching. We recall that quenching is the rapid cooling of a hot object in the air or in a liquid such as water or oil to obtain certain properties. The study of quenching phenomena began in 1975 with an article by Kawarada [15], where he studied a model in one space dimension. This article initiated a broad study of the quenching problem by many scientific researchers, including work on existence, the structure of quenching points, the asymptotic behaviour of solutions, etc. Since then, this notion has been widely studied and developed in hundreds of books and scientific articles. For a detailed survey, we refer also to Levine [20].

The quenching phenomena have been studied by many researchers and therefore we find a lot of works in this field. This is due to its many and varied applications in the fields of science, especially engineering. We find a lot of models in Constantin et al. [6], Kiselev and Zlatos [16] and Marion [22]. For example, quenching can increase the hardness of metallic or plastic materials, to see other examples we refer to Aris [3], Muntean [26] and references given there. In this kind of problems, singularities can appear in the solutions of the systems formulated. This can complicate the theoretical and numerical mathematical analysis of the problem.

In this work, we are interested in the study of a singular reaction diffusion system. For modeling and mathematical analysis of this type of problem, several methods have been

Email address: salimbra@gmail.com

Received: 11.05.2021; Accepted: 22.01.2022 
proposed; see for example, [1,2], [4], [5], [7], [9-14], [21-24], [29-33], [37] and corresponding references therein.

The study of specialized behaviour for reaction diffusion systems has been an active area of research for decades. Two types of specialized behaviour, solution explosion and solution quenching, have been of particular interest more recently. In explosion problems, the solution becomes unbounded in finite time, i.e. when $t$ approaches the explosion instant $T$, which is finite. Naturally, the reason for this behaviour of the solution is due to the singularity of the nonlinearities. In quenching problems, the solution remains bounded while the first order time derivative becomes unbounded in finite time. We refer also to Salin's works [30-33], Selçuk [34] and to references therein for more detailed information and a mathematical analysis of models involving singular terms.

So, the objective here is the study of a notion which one calls "quenching" generalizing that of the explosion. We are then interested in the study of the existence of weak solutions of the following reaction diffusion system with singular nonlinearities :

$$
\begin{cases}\frac{\partial u}{\partial t}-\Delta u=F(t, x, u, v) & \text { in } Q_{T} \\ \frac{\partial v}{\partial t}-\Delta v=G(t, x, u, v) & \text { in } Q_{T}\end{cases}
$$

with the initial conditions

$$
\begin{cases}u(0, x)=u_{0}(x) & \text { in } \Omega \\ v(0, x)=v_{0}(x) & \text { in } \Omega\end{cases}
$$

and the following boundary conditions

$$
\begin{cases}u(t, x)=0 & \text { on } \Gamma_{1} \times(0, T) \\ v(t, x)=0 & \text { on } \Gamma_{2} \times(0, T) \\ \frac{\partial u}{\partial \nu}=0 & \text { on } \Gamma_{2} \times(0, T) \\ \frac{\partial v}{\partial \nu}=0 & \text { on } \Gamma_{1} \times(0, T)\end{cases}
$$

with

$$
\left\{\begin{array}{l}
F(t, x, r, s)=f(t, x) \frac{s}{r^{\gamma}} \\
G(t, x, r, s)=g(t, x) \frac{s}{r^{\gamma}}
\end{array}\right.
$$

where $Q_{T}=\Omega \times(0, T), \Omega$ is a bounded Lipschitz domain of $\mathbb{R}^{N}, N \geq 2$ and $T>0 . \gamma$ is a real parameter such as $0<\gamma \leq 1$. $\Gamma_{1}$ and $\Gamma_{2}$ are such that $\Gamma_{1} \cup \Gamma_{2}=\partial \Omega$ and $\Gamma_{1} \cap \Gamma_{2}=\phi$. The Haussdorff measure of $\Gamma_{1}$ and $\Gamma_{2}$ does not vanish. Here $\nu$ denotes the outer normal to $\partial \Omega$. The functions $f, g: Q_{T} \rightarrow \mathbb{R}$ and satisfy the following conditions :

$$
f, g \in L^{1}\left(Q_{T}\right) \quad, \quad f \geq 0 \text { and } f+g \leq 0
$$

Note that the functions $F$ and $G$ are singular at $r=0$, and we assume that the functions $u_{0}$ and $v_{0}$ are such that

$$
u_{0}, v_{0} \geq 0 \text { and } u_{0}, v_{0} \in L^{\infty}(\Omega)
$$

We will study our problem in the following spaces :

$$
\begin{gathered}
V=\left\{\varphi \in H^{1}(\Omega): \varphi=0 \quad \text { on } \Gamma_{1}\right\} \\
W=\left\{\psi \in H^{1}(\Omega): \psi=0 \quad \text { on } \Gamma_{2}\right\}
\end{gathered}
$$

The dual spaces of $V$ and $W$, respectively, are denoted by $V^{*}$ and $W^{*}$.

The problem $(1.1)-(1.4)$ consists of a weakly coupled system whose right term is singular in the variable $u$. We hear that the nonlinearities $F(t, x, r, s)$ and $G(t, x, r, s)$ can become unbounded to the neighborhood of $r=0$. 
The main contribution is to study a reaction diffusion system with singular and nonregular nonlinearities $F$ and $G$ dependent on the independent variables $t$ and $x$ and on the unknown functions $u$ and $v$. The functions $f$ and $g$ verify simple properties, this allows us to choose them from a wide range.

We confirm that the studied model is not only applied to the quenching phenomenon, but can also be applied to other singular reaction diffusion models in different scientific fields.

Before stating the main result of this work, it is worth mentioning that several mathematicians have dealt with this type of problem using various analytical and numerical techniques and methods, under different hypotheses as appropriate, see for example, [4], [7], [9-14] and [37].

This document is organized as follows : In the next section, we state our main result. In the third section, we give a result concerning the nonsingular approximating problem. In the fourth section, we give important a priori estimates. The fifth section is devoted to some important results of convergence and compactness. In the sixth section, we prove certain properties relating to our problem near the singularity. The last section is devoted to prove the main result. The paper ends with a conclusion and some perspectives.

The difficulties in this work are similar to those in [9-14], and the techniques are of the same spirit, but specific new difficulties due to the nature of the system must be handled.

\section{The main result}

We first introduce the notion of weak solution to the problem (1.1) - (1.4) used here.

Definition 2.1. A weak solution to problem (1.1) - (1.4) is a nonnegative couple

$$
(u, v) \in\left[L^{2}(0, T ; V) \cap L^{\infty}\left(0, T ; L^{2}(\Omega)\right] \times\left[L^{2}(0, T ; W) \cap L^{\infty}\left(0, T ; L^{2}(\Omega)\right)\right]\right.
$$

with

$$
\left(u_{t}, v_{t}\right) \in\left[L^{2}\left(0, T ; V^{*}\right)+L^{1}\left(0, T ; L_{l o c}^{1}(\Omega)\right)\right] \times\left[L^{2}\left(0, T ; W^{*}\right)+L^{1}\left(0, T ; L_{l o c}^{1}(\Omega)\right)\right]
$$

such that

$$
\begin{gathered}
u(0, x)=u_{0}(x), v(0, x)=v_{0}(x) \quad \text { a.e. } x \in \Omega \\
\int_{Q_{T}} f \frac{v}{u^{\gamma}} \phi<+\infty, \quad \int_{Q_{T}} g \frac{v}{u^{\gamma}} \eta<+\infty
\end{gathered}
$$

and

$$
\begin{aligned}
-\int_{\Omega} u_{0}(x) \varphi(0, x)-\int_{Q_{T}} u \frac{\partial \varphi}{\partial t}+\int_{Q_{T}} \nabla u \nabla \varphi & =\int_{Q_{T}} F \varphi \\
-\int_{\Omega} v_{0}(x) \psi(0, x)-\int_{Q_{T}} v \frac{\partial \psi}{\partial t}+\int_{Q_{T}} \nabla v \nabla \psi & =\int_{Q_{T}} G \psi
\end{aligned}
$$

for all $\phi, \eta, \varphi, \psi \in C_{0}^{\infty}([0, T) \times \Omega)$.

Now, we can state the main result of this work :

Theorem 2.2. Assume (1.4) - (1.6), then there exists a weak solution $(u, v)$ to problem $(1.1)-(1.4)$ in the sense of Definition 2.1.

\section{Nonsingular approximating problem}

To study the problem (1.1) - (1.4), we consider the nonsingular approximating problem. Essentially, we are truncating in such a way as to eliminate the singularity. In this case, we define the sequences of functions $f_{n}$ and $g_{n}$, such that

$$
\begin{aligned}
& f_{n}(t, x)=\min \{n, f(t, x)\} \\
& g_{n}(t, x)=-\min \{n,-g(t, x)\}
\end{aligned}
$$


It is easily seen that $f_{n}$ and $g_{n}$ satisfy the same properties as $f$ and $g$, moreover,

$$
\begin{array}{ll}
0 \leq f_{n} \leq f & , \quad \lim _{n \rightarrow+\infty} f_{n}=f \\
g \leq g_{n} \leq 0 & , \quad \lim _{n \rightarrow+\infty} g_{n}=g
\end{array}
$$

The approximate problem is the following :

$$
\text { Find }\left(u_{n}, v_{n}\right) \text { in }\left[L^{2}(0, T ; V) \cap L^{\infty}\left(Q_{T}\right)\right] \times\left[L^{2}(0, T ; W) \cap L^{\infty}\left(Q_{T}\right)\right]
$$

such that

$$
\begin{cases}\left(u_{n}\right)_{t}-\Delta u_{n}=F_{n}\left(t, x, u_{n}, v_{n}\right) & \text { in } Q_{T} \\ \left(v_{n}\right)_{t}-\Delta v_{n}=G_{n}\left(t, x, u_{n}, v_{n}\right) & \text { in } Q_{T} \\ u_{n}(0, x)=u_{0, n}(x) & \text { in } \Omega \\ v_{n}(0, x)=v_{0, n}(x) & \text { in } \Omega \\ u_{n}(t, x)=0 & \text { on } \Gamma_{1} \times(0, T) \\ v_{n}(t, x)=0 & \text { on } \Gamma_{2} \times(0, T) \\ \frac{\partial u_{n}}{\partial \nu}=0 & \text { on } \Gamma_{2} \times(0, T) \\ \frac{\partial v_{n}}{\partial \nu}=0 & \text { on } \Gamma_{1} \times(0, T)\end{cases}
$$

where

$$
\begin{aligned}
& F_{n}\left(t, x, u_{n}, v_{n}\right)= \begin{cases}f_{n}(t, x) \frac{v_{n}}{\left(u_{n}+\frac{1}{n}\right)^{\gamma}} & \text { if } u_{n} \geq 0 \text { and } v_{n} \geq 0 \\
0 & \text { otherwise }\end{cases} \\
& G_{n}\left(t, x, u_{n}, v_{n}\right)= \begin{cases}g_{n}(t, x) \frac{v_{n}}{\left(u_{n}+\frac{1}{n}\right)^{\gamma}} & \text { if } u_{n} \geq 0 \text { and } v_{n} \geq 0 \\
0 & \text { otherwise }\end{cases}
\end{aligned}
$$

while $u_{0, n}, v_{0, n} \in L^{\infty}(\Omega) \cap H_{0}^{1}(\Omega)$ are suitable regularizations of the initial data obtained by a standard convolution technique (see [8]) such that

$$
\begin{aligned}
& \lim _{n \rightarrow \infty} \frac{1}{n}\left\|u_{0, n}\right\|_{H_{0}^{1}(\Omega)}=0 \\
& \lim _{n \rightarrow \infty} \frac{1}{n}\left\|v_{0, n}\right\|_{H_{0}^{1}(\Omega)}=0
\end{aligned}
$$

We have the following important result :

Lemma 3.1. Problem (3.1) admits a nonnegative couple of solutions :

$$
\left(u_{n}, v_{n}\right) \in\left[L^{2}(0, T ; V) \cap L^{\infty}\left(Q_{T}\right)\right] \times\left[L^{2}(0, T ; W) \cap L^{\infty}\left(Q_{T}\right)\right]
$$

such that

$$
\begin{aligned}
& -\int_{\Omega} u_{0, n}(x) \varphi(0, x)-\int_{Q_{T}} u_{n} \frac{\partial \varphi}{\partial t}+\int_{Q_{T}} \nabla u_{n} \nabla \varphi=\int_{Q_{T}} F_{n}\left(t, x, u_{n}, v_{n}\right) \varphi \\
& -\int_{\Omega} v_{0, n}(x) \psi(0, x)-\int_{Q_{T}} v_{n} \frac{\partial \psi}{\partial t}+\int_{Q_{T}} \nabla v_{n} \nabla \psi=\int_{Q_{T}} G_{n}\left(t, x, u_{n}, v_{n}\right) \psi
\end{aligned}
$$

for every $\varphi, \psi \in C_{0}^{\infty}(\Omega \times[0, T))$.

Proof. For simplicity, we suppose $u_{0, n}=0$ and $v_{0, n}=0$. Then, by a direct application of the method of Stampacchia in [36], we can prove the positivity of the solution by taking as test function in the first equation of the problem (3.1) the function $\varphi=-u_{n}^{-}$, where

$$
u_{n}=u_{n}^{+}-u_{n}^{-}, u_{n}^{+}=\max \left\{u_{n}, 0\right\} \quad, u_{n}^{-}=\max \left\{-u_{n}, 0\right\}
$$


Since $u_{n}^{+}=0$ on the support of $u_{n}^{-}$, we have that the right hand side of (3.4) is zero because

$$
F_{n}\left(t, x, u_{n}, v_{n}\right)= \begin{cases}f_{n}(t, x) \frac{v_{n}}{\left(u_{n}+\frac{1}{n}\right)^{\gamma}} & \text { if } u_{n} \geq 0 \text { and } v_{n} \geq 0 \\ 0 & \text { otherwise }\end{cases}
$$

so we have

$$
\int_{Q_{T}}\left(u_{n}\right)_{t}\left(-u_{n}^{-}\right)+\int_{Q_{T}} \nabla u_{n} \nabla\left(-u_{n}^{-}\right)=0
$$

which give

$$
\int_{Q_{T}}\left(u_{n}^{+}-u_{n}^{-}\right)_{t}\left(-u_{n}^{-}\right)+\int_{Q_{T}} \nabla\left(u_{n}^{+}-u_{n}^{-}\right) \nabla\left(-u_{n}^{-}\right)=0
$$

We observe that on the support of $u_{n}^{-}$we have $u_{n}^{+} \cdot u_{n}^{-}=0$, he comes

$$
\frac{1}{2} \int_{\Omega}\left(u_{n}^{-}\right)^{2}(t)+\int_{Q_{\tau}}\left|\nabla\left(u_{n}^{-}\right)\right|^{2}=0, \text { for all } t \in[0, T]
$$

and we deduce that

$$
u_{n}^{-}=0 \quad \text { a.e. in } Q_{T}
$$

i.e. that $u_{n} \geq 0$ a.e. in $\Omega$ and for all $t \in[0, T)$. In the same way, we prove that $v_{n} \geq 0$, by choosing as test function $\psi=-v_{n}^{-}$.

In everything that follows, we denote with $C$ a generic constant. Usually $C$ is thought to be independent of $n$, if not otherwise mentioned. Before giving the proof of our result, let us denote by $T_{k}$ the truncation function

$$
T_{k}(s)=\max \{-k, \min \{k, s\}\}, \quad k \geq 0, s \in \mathbb{R}
$$

and by $\mathcal{G}_{k}$ the function

$$
\mathcal{G}_{k}(s)=s-T_{k}(s)
$$

In the following, we will denote by $\langle\cdot, \cdot\rangle$ the duality product between $V^{*}$ and $V$ (and also between $W^{*}$ and $W$ ).

\section{A priori uniform estimates}

\subsection{Uniform estimate for $\left(u_{n}, v_{n}\right)$ in $L^{\infty}\left(Q_{T}\right)$}

Proposition 4.1. There exist positive constants $M_{1}$ and $M_{2}$, independent of $n$, such that

$$
\begin{aligned}
\left\|u_{n}\right\|_{L^{\infty}\left(Q_{T}\right)} & \leq M_{1} \\
\left\|v_{n}\right\|_{L^{\infty}\left(Q_{T}\right)} & \leq M_{2}
\end{aligned}
$$

Proof. The uniform estimate (4.1) for the sequence $\left\{u_{n}\right\}$ follows directly by Proposition 2.13 in [11] with some abbreviations that go along with our problem. For simplicity we suppose $v_{0, n}(x)=0$. To handle the equation solved by $v_{n}$ we choose as test function

$$
\psi=\mathcal{G}_{M_{2}}\left(v_{n}\right):=\left(v_{n}-M_{2}\right)^{+}
$$

with $M_{2}>1$ fixed, we obtain, with $Q_{t}:=\Omega \times[0, t)$

$$
\begin{aligned}
& \int_{Q_{t}}\left(v_{n}\right)_{t}\left(v_{n}-M_{2}\right)^{+}+\int_{Q_{t}} \nabla v_{n} \cdot \nabla\left(v_{n}-M_{2}\right)^{+} \\
= & \int_{Q_{t}} g_{n}(t, x) \frac{v_{n}}{\left(u_{n}+\frac{1}{n}\right)^{\gamma}}\left(v_{n}-M_{2}\right)^{+} \leq 0
\end{aligned}
$$

Neglecting the nonnegative term on the left hand side, it comes

$$
\left(v_{n}-M_{2}\right)^{+}=0 \text { a.e. in } Q_{T}
$$

which proves (4.2). 
4.2. Energy estimate for $\left(u_{n}, v_{n}\right)$ in $L^{2}(0, T ; V) \times L^{2}(0, T ; W)$

Proposition 4.2. There exists a positive constant $C$, independent of $n$, such that

$$
\left\|u_{n}\right\|_{L^{2}(0, T ; V)}+\left\|v_{n}\right\|_{L^{2}(0, T ; W)} \leq C
$$

Proof. Choosing as test function $\varphi=u_{n} \in L^{2}(0, T ; V)$ in the first equation of problem (3.1) solved by $u_{n}$ and integrating over $\Omega \times[0, t)$, we obtain

$$
\frac{1}{2} \frac{d}{d t} \int_{Q_{T}} u_{n}^{2} d t+\int_{Q_{T}}\left|\nabla u_{n}\right|^{2}=\int_{Q_{T}} f_{n}(t, x) \frac{v_{n} u_{n}}{\left(u_{n}+\frac{1}{n}\right)^{\gamma}}
$$

which give

$$
\frac{1}{2} \int_{\Omega} u_{n}^{2}(t) d t+\int_{Q_{T}}\left|\nabla u_{n}\right|^{2}=\frac{1}{2}\left\|u_{0, n}\right\|_{L^{2}(\Omega)}^{2}+\int_{Q_{T}} f_{n}(t, x) \frac{v_{n} u_{n}}{\left(u_{n}+\frac{1}{n}\right)^{\gamma}}
$$

By observing that $\frac{u_{n}}{\left(u_{n}+\frac{1}{n}\right)^{\gamma}} \leq u_{n}^{1-\gamma}$ and $0<1-\gamma<1$, we obtain

$$
\int_{Q_{T}} f_{n}(t, x) \frac{v_{n} u_{n}}{\left(u_{n}+\frac{1}{n}\right)^{\gamma}} \leq \int_{Q_{T}} f_{n}(t, x) u_{n}^{1-\gamma} v_{n} \leq \sup \left|u_{n}^{1-\gamma} v_{n}\right| \int_{Q_{T}} f_{n} \leq C
$$

We then obtain

$$
\left\|u_{n}\right\|_{L^{\infty}\left(0, T ; L^{2}(\Omega)\right)} \leq C
$$

For the second equation of problem (3.1), we choose as test function $\psi=v_{n} \in L^{2}(0, T ; W)$. We obtain

$$
\begin{aligned}
\frac{1}{2} \int_{\Omega} v_{n}^{2}(t) d t+\int_{Q_{T}}\left|\nabla v_{n}\right|^{2} & =\frac{1}{2}\left\|v_{0, n}\right\|_{L^{2}(\Omega)}^{2}+\int_{Q_{T}} g_{n}(t, x) \frac{v_{n} u_{n}}{\left(u_{n}+\frac{1}{n}\right)^{\gamma}} \\
& \leq \frac{1}{2}\left\|v_{0, n}\right\|_{L^{2}(\Omega)}^{2}
\end{aligned}
$$

which give

hence the inequality (4.3).

$$
\left\|v_{n}\right\|_{L^{\infty}\left(0, T ; L^{2}(\Omega)\right)} \leq C
$$

Proposition 4.3. There exists a positive constant $C$, independent of $n$, such that

$$
\begin{aligned}
& \int_{Q_{T}} f_{n}(t, x) \frac{v_{n}}{\left(u_{n}+\frac{1}{n}\right)^{\gamma}} \varphi^{2}(x) \leq C, \quad \text { for all } n \in \mathbb{N} \\
& \int_{Q_{T}}\left|g_{n}(t, x)\right| \frac{v_{n}}{\left(u_{n}+\frac{1}{n}\right)^{\gamma}} \psi^{2}(x) \leq C, \quad \text { for all } n \in \mathbb{N}
\end{aligned}
$$

for every $\varphi, \psi \in C_{0}^{\infty}(\Omega)$.

Proof. (i) We multiply the first equation of problem (3.1) by the test function $\varphi^{2}(x)$, we get

$$
\int_{0}^{T}\left\langle\left(u_{n}\right)_{t}, \varphi^{2}(x)\right\rangle+2 \int_{Q_{T}} \nabla u_{n} \varphi \nabla \varphi=\int_{Q_{T}} f_{n}(t, x) \frac{v_{n}}{\left(u_{n}+\frac{1}{n}\right)^{\gamma}} \varphi^{2}(x)
$$

which give

$$
\int_{Q_{T}} f_{n}(t, x) \frac{v_{n}}{\left(u_{n}+\frac{1}{n}\right)^{\gamma}} \varphi^{2}(x) \leq 2 \int_{Q_{T}}\left|\nabla u_{n}\right| \cdot|\varphi| \cdot|\nabla \varphi|+C
$$

this gives, by applying the Hölder's inequality and the previous Proposition

$$
\int_{Q_{T}} f_{n}(t, x) \frac{v_{n}}{\left(u_{n}+\frac{1}{n}\right)^{\gamma}} \varphi^{2}(x) \leq C+2\left(\int_{Q_{T}}\left|\nabla u_{n}\right|^{2} \cdot \varphi^{2}\right)^{\frac{1}{2}} \cdot\left(\int_{Q_{T}}|\nabla \varphi|^{2}\right)^{\frac{1}{2}} \leq C_{2}
$$


which proves the inequality (4.4).

(ii) In the same way, but this time we multiply the second equation of problem (3.1) by the test function $\psi^{2}(x)$, we get

$$
\int_{0}^{T}\left\langle\left(v_{n}\right)_{t}, \psi^{2}(x)\right\rangle+2 \int_{Q_{T}} \nabla v_{n} \psi \nabla \psi=\int_{Q_{T}} g_{n}(t, x) \frac{v_{n}}{\left(u_{n}+\frac{1}{n}\right)^{\gamma}} \psi^{2}(x)
$$

which give

$$
\left|\int_{0}^{T}\left\langle\left(v_{n}\right)_{t}, \psi^{2}(x)\right\rangle\right|+2\left|\int_{Q_{T}} \nabla v_{n} \psi \nabla \psi\right| \geq\left|\int_{Q_{T}} g_{n}(t, x) \frac{v_{n}}{\left(u_{n}+\frac{1}{n}\right)^{\gamma}} \psi^{2}(x)\right|
$$

We choose $C$ so that

$$
\int_{Q_{T}}\left|g_{n}(t, x)\right| \frac{v_{n}}{\left(u_{n}+\frac{1}{n}\right)^{\gamma}} \psi^{2}(x) \leq C+2 \int_{Q_{T}}\left|\nabla v_{n}\right| \cdot|\psi| \cdot|\nabla \psi|
$$

this gives, by applying the Hölder's inequality and the previous Proposition

$$
\int_{Q_{T}}\left|g_{n}(t, x)\right| \frac{v_{n}}{\left(u_{n}+\frac{1}{n}\right)^{\gamma}} \psi^{2}(x) \leq C+2\left(\int_{Q_{T}}\left|\nabla v_{n}\right|^{2} \cdot \psi^{2}\right)^{\frac{1}{2}} \cdot\left(\int_{Q_{T}}|\nabla \psi|^{2}\right)^{\frac{1}{2}} \leq C_{2}
$$

which gives the desired result (4.5).

\subsection{Uniform estimate on critical sets}

In this paragraph we will consider the following critical sets

$$
\left\{(t, x) \in Q_{T}: u_{n}(t, x) \leq \delta\right\} \text { and }\left\{(t, x) \in Q_{T}: v_{n}(t, x) \leq \delta\right\}
$$

These sets are prone to hosting the locations of the singularity. In fact, we wish to avoid a potential blow up of the solutions on these sets. This is ensured by the following Proposition :

Proposition 4.4. For $\gamma>0$, we have

$$
\begin{gathered}
\int_{Q_{T} \cap\left\{0 \leq u_{n} \leq \delta\right\}} f_{n}(t, x) \frac{v_{n}}{\left(u_{n}+\frac{1}{n}\right)^{\gamma}} \varphi^{2}(x) \leq C \delta \\
\int_{Q_{T} \cap\left\{0 \leq v_{n} \leq \delta\right\}} f_{n}(t, x) \frac{v_{n}}{\left(u_{n}+\frac{1}{n}\right)^{\gamma}} \varphi^{2}(x) \leq \begin{cases}C \delta^{1-\gamma} & \text { if } 0<\gamma<1 \\
C \sqrt{\delta} & \text { if } \gamma=1\end{cases} \\
\int_{Q_{T} \cap\left\{0 \leq v_{n} \leq \delta\right\}}\left|g_{n}(t, x)\right| \frac{v_{n}}{\left(u_{n}+\frac{1}{n}\right)^{\gamma}} \varphi^{2}(x) \leq C \delta \\
\int_{Q_{T} \cap\left\{0 \leq u_{n} \leq \delta\right\}}\left|g_{n}(t, x)\right| \frac{v_{n}}{\left(u_{n}+\frac{1}{n}\right)^{\gamma}} \varphi^{2}(x) \leq \begin{cases}C \delta^{1-\gamma} & \text { if } 0<\gamma<1 \\
C \sqrt{\delta} & \text { if } \gamma=1\end{cases}
\end{gathered}
$$

for every $\varphi \in C_{0}^{\infty}(\Omega)$ with $\varphi \geq 0$.

Proof. (i) We prove first (4.6). Following the same ideas as in the proof of Proposition 2.20 in [11], we choose as test function in the equation solved by $u_{n}$ the function $\varphi_{\sigma}=$ 
$\frac{T_{\sigma}\left(-\left(u_{n}-\delta\right)^{-}\right)}{\sigma} \varphi^{2}(x)$, with $\varphi \in C_{0}^{\infty}(\Omega)$ and $\varphi \geq 0$. Therefore we get

$$
\begin{gathered}
\int_{0}^{T}\left\langle\left(u_{n}\right)_{t}, \frac{T_{\sigma}\left(-\left(u_{n}-\delta\right)^{-}\right)}{\sigma} \varphi^{2}(x)\right\rangle+\frac{1}{\sigma} \int_{Q_{T}} \nabla u_{n} \nabla\left(T_{\sigma}\left(-\left(u_{n}-\delta\right)^{-}\right)\right) \varphi^{2}(x) \\
+2 \int_{Q_{T}} \nabla u_{n} \frac{T_{\sigma}\left(-\left(u_{n}-\delta\right)^{-}\right)}{\sigma} \varphi \nabla \varphi \\
=\int_{Q_{T}} f_{n}(t, x) \frac{v_{n}}{\left(u_{n}+\frac{1}{n}\right)^{\gamma}} \frac{\frac{T}{\sigma}\left(-\left(u_{n}-\delta\right)^{-}\right)}{\sigma} \varphi^{2}(x)
\end{gathered}
$$

First, we show that

$$
\int_{0}^{T}\left\langle\left(u_{n}\right)_{t}, \frac{T_{\sigma}\left(-\left(u_{n}-\delta\right)^{-}\right)}{\sigma} \varphi^{2}(x)\right\rangle \geq-\delta|\Omega|
$$

where $|\Omega|$ is the Lebesgue measure of $\Omega$. For that, we introduce the function $v_{\sigma, \nu}=$ $\frac{T_{\sigma}\left(-\left(u_{n, \nu}-\delta\right)^{-}\right)}{\sigma}$, where $u_{n, \nu}$ is, for any fixed $n \in \mathbb{N}$ and $\sigma \in \mathbb{N}$, the solution of the following ordinary differential equation problem

$$
\left\{\begin{array}{l}
\frac{1}{\sigma}\left[u_{n, \nu}\right]_{t}+u_{n, \nu}=u_{n} \\
u_{n, \nu}(0)=u_{0, n}
\end{array}\right.
$$

The function $u_{n, \nu}$ satisfies the following properties (see [17] and [18]) :

$$
\begin{gathered}
u_{n, \nu} \in L^{2}\left(0, T ; H_{0}^{1}(\Omega)\right), \quad\left(u_{n, \nu}\right)_{t} \in L^{2}\left(0, T ; H_{0}^{1}(\Omega)\right) \\
\left\|u_{n, \nu}\right\|_{L^{\infty}\left(Q_{T}\right)} \leq\left\|u_{n}\right\|_{L^{\infty}\left(Q_{T}\right)} \\
u_{n, \nu} \rightarrow u_{n} \quad \text { in } L^{2}\left(0, T ; H_{0}^{1}(\Omega)\right) \quad \text { as } \nu \rightarrow+\infty \\
\left(u_{n, \nu}\right)_{t} \rightarrow\left(u_{n}\right)_{t} \quad \text { in } L^{2}\left(0, T ; H_{0}^{-1}(\Omega)\right) \text { as } \nu \rightarrow+\infty
\end{gathered}
$$

So, we have

$$
\begin{gathered}
\int_{0}^{T}\left\langle\left(u_{n}\right)_{t}, \frac{T_{\sigma}\left(-\left(u_{n}-\delta\right)^{-}\right)}{\sigma} \varphi^{2}(x)\right\rangle \\
=\lim _{\nu \rightarrow \infty} \int_{Q_{T}}\left[\left(u_{n, \nu}-\delta\right)_{t}^{+}\right] \frac{T_{\sigma}\left(-\left(u_{n, \nu}-\delta\right)^{-}\right)}{\sigma} \varphi^{2}(x) \\
-\lim _{\nu \rightarrow \infty} \int_{Q_{T}}\left[\left(u_{n, \nu}-\delta\right)_{t}^{-}\right] \frac{T_{\sigma}\left(-\left(u_{n, \nu}-\delta\right)^{-}\right)}{\sigma} \varphi^{2}(x) \\
=\lim _{\nu \rightarrow \infty} \int_{Q_{T}}\left[\left(u_{n, \nu}-\delta\right)_{t}^{-}\right] \frac{T_{\sigma}\left(\left(u_{n, \nu}-\delta\right)^{-}\right)}{\sigma} \varphi^{2}(x)
\end{gathered}
$$

Introducing now the function $\Phi_{\sigma}(s):=\int_{0}^{(s-\delta)^{-}} \frac{T_{\sigma}(\rho)}{\sigma} d \rho$, from (4.13), we obtain

$$
\begin{aligned}
& \lim _{\nu \rightarrow \infty} \int_{Q_{T}}\left(u_{n, \nu}-\delta\right)_{t}^{-} \frac{T_{\sigma}\left(\left(u_{n, \nu}-\delta\right)^{-}\right)}{\delta} \varphi^{2}(x) \\
& =\lim _{\nu \rightarrow \infty} \int_{Q_{T}} \frac{d}{d t} \Phi_{\sigma}\left(u_{n, \nu}\right) \\
& =\lim _{\nu \rightarrow \infty} \int_{\Omega} \Phi_{\sigma}\left(u_{n, \nu}-\delta\right)^{-}(T)-\lim _{\nu \rightarrow \infty} \int_{\Omega} \Phi_{\sigma}\left(u_{n, \nu}-\delta\right)^{-}(0) \\
& \geq-\lim _{\nu \rightarrow \infty} \int_{\Omega} \Phi_{\sigma}\left(u_{n, \nu}-\delta\right)^{-}(0)=-\int_{\Omega} \Phi_{\sigma}\left(u_{n}-\delta\right)^{-}(0) \geq-\delta|\Omega|
\end{aligned}
$$


which proves (4.11). By (4.11), observing also that $\frac{T_{\sigma}\left(-\left(u_{n}-\delta\right)^{-}\right)}{\sigma}=0$ on the set $\{(x, t) \in$ $\left.Q_{T}: u_{n}(x, t) \geq \delta\right\}$, the equality (4.10) becomes

$$
\begin{gathered}
\frac{1}{\sigma} \int_{Q_{T} \cap\left\{\delta-\sigma \leq u_{n} \leq \delta\right\}}\left|\nabla u_{n}\right|^{2} \cdot \varphi^{2}(x)+\int_{Q_{T}} f_{n}(t, x) \frac{v_{n}}{\left(u_{n}+\frac{1}{n}\right)^{\gamma}} \frac{T_{\sigma}\left(\left(u_{n, \nu}-\delta\right)^{-}\right)}{\sigma} \varphi^{2}(x) \\
\leq 2 \int_{Q_{T} \cap\left\{u_{n} \leq \delta\right\}}\left|\nabla u_{n}\right| \cdot \varphi \cdot|\nabla \varphi|+\delta|\Omega|
\end{gathered}
$$

Using Hölder's inequality in the right hand side of (4.14), we obtain

$$
\begin{aligned}
& \int_{Q_{T}} f_{n}(t, x) \frac{v_{n}}{\left(u_{n}+\frac{1}{n}\right)^{\gamma}} \frac{T_{\sigma}\left(\left(u_{n, \nu}-\delta\right)^{-}\right)}{\sigma} \varphi^{2}(x) \\
\leq & 2\left(\int_{Q_{T} \cap\left\{u_{n} \leq \delta\right\}}\left|\nabla u_{n}\right|^{2} \cdot \varphi^{2}\right)^{\frac{1}{2}}\left(\int_{Q_{T}}|\nabla \varphi|^{2}\right)^{\frac{1}{2}}+\delta|\Omega|
\end{aligned}
$$

Now, we can prove that

$$
\int_{Q_{T} \cap\left\{u_{n} \leq \delta\right\}}\left|\nabla u_{n}\right|^{2} \varphi^{2}(x) \leq C \delta
$$

Indeed, multiplying problem (3.1) by the test function $-\left(u_{n}-\delta\right)^{-} \varphi^{2}(x), \varphi \in C_{0}^{\infty}(\Omega)$, $\varphi \geq 0$, we obtain

$$
\begin{gathered}
\int_{0}^{T}\left\langle\left(u_{n}\right)_{t},\left(-\left(u_{n}-\delta\right)^{-}\right) \varphi^{2}\right\rangle+ \\
\int_{Q_{T} \cap\left\{u_{n} \leq \delta\right\}}\left|\nabla u_{n}\right|^{2} \varphi^{2}-2 \int_{Q_{T}} \nabla u_{n}\left(u_{n}-\delta\right)^{-} \varphi \nabla \varphi \leq 0
\end{gathered}
$$

For the first term of (4.16), we follow the same arguments as those used to obtain (4.11), he comes

$$
\int_{0}^{T}\left\langle\left(u_{n}\right)_{t},\left(-\left(u_{n}-\delta\right)^{-}\right) \varphi^{2}(x)\right\rangle \geq-\delta|\Omega|
$$

By (4.17), the inequality (4.16) becomes

$$
\int_{Q_{T} \cap\left\{u_{n} \leq \delta\right\}}\left|\nabla u_{n}\right|^{2} \varphi^{2} \leq 2 \int_{Q_{T} \cap\left\{u_{n}<\delta\right\}}\left|\nabla u_{n}\right|\left(\delta-u_{n}\right) \varphi|\nabla \varphi|+\delta|\Omega|
$$

which, by Hölder's inequality and (4.3), leads to

$$
\begin{aligned}
\int_{Q_{T} \cap\left\{u_{n} \leq \delta\right\}}\left|\nabla u_{n}\right|^{2} \varphi^{2} & \leq 2 \delta\left(\int_{Q_{T}}\left|\nabla u_{n}\right|^{2} \varphi^{2}\right)^{\frac{1}{2}}\left(\int_{Q_{T}}|\nabla \varphi|^{2}\right)^{\frac{1}{2}}+\delta|\Omega| \\
& \leq C \delta
\end{aligned}
$$

Thus, (4.15) holds. Finally, we have obtained that

$$
\int_{Q_{T}} f_{n}(t, x) \frac{v_{n}}{\left(u_{n}+\frac{1}{n}\right)^{\gamma}} \frac{T_{\sigma}\left(\left(u_{n, \nu}-\delta\right)^{-}\right)}{\delta} \varphi^{2}(x) \leq C \delta
$$

Now, we can pass to the limit in (4.18) for $\sigma \rightarrow 0$ and $n$ fixed. For this we use Lebesgue dominate convergence Theorem since $\frac{T_{\sigma}\left(\left(u_{n}-\delta\right)^{-}\right)}{\sigma} \rightarrow 1$ a.e. on the set $\left\{(x, t) \in Q_{T}\right.$ : $\left.u_{n}(t, x)<\delta\right\}$. Therefore, we obtain

$$
\int_{Q_{T} \cap\left\{0 \leq u_{n} \leq \delta\right\}} f_{n}(t, x) \frac{v_{n}}{\left(u_{n}+\frac{1}{n}\right)^{\gamma}} \varphi^{2} \leq C \delta
$$

and hence (4.6) holds. 
(ii) We are now focusing on estimation (4.7). If $0<\gamma<1$, we consider the decomposition

$$
\begin{gathered}
\int_{Q_{T} \cap\left\{0 \leq v_{n} \leq \delta\right\}} f_{n}(t, x) \frac{v_{n}}{\left(u_{n}+\frac{1}{n}\right)^{\gamma}} \varphi^{2}(x) \\
=\int_{Q_{T} \cap\left\{0 \leq v_{n} \leq \delta\right\} \cap\left\{0 \leq u_{n} \leq \delta\right\}} f_{n}(t, x) \frac{v_{n}}{\left(u_{n}+\frac{1}{n}\right)^{\gamma}} \varphi^{2}(x) \\
+\int_{Q_{T} \cap\left\{0 \leq v_{n} \leq \delta\right\} \cap\left\{u_{n}>\delta\right\}} f_{n}(t, x) \frac{v_{n}}{\left(u_{n}+\frac{1}{n}\right)^{\gamma}} \varphi^{2}(x) \\
\leq \int_{Q_{T} \cap\left\{0 \leq u_{n} \leq \delta\right\}} f_{n}(t, x) \frac{v_{n}}{\left(u_{n}+\frac{1}{n}\right)^{\gamma}} \varphi^{2}(x) \\
+\int_{Q_{T} \cap\left\{0 \leq v_{n} \leq \delta\right\} \cap\left\{u_{n}>\delta\right\}} f_{n}(t, x) \frac{v_{n}}{\left(u_{n}+\frac{1}{n}\right)^{\gamma}} \varphi^{2}(x) \\
=I_{1}+I_{2}
\end{gathered}
$$

By (4.6), we obtain

$$
I_{1} \leq C \delta
$$

To handle the term $I_{2}$, we proceed as follows : Since $v_{n} \leq \delta$ and $\left(\frac{1}{n}+u_{n}\right)^{\gamma}>\delta^{\gamma}$, he comes $\frac{1}{\left(u_{n}+\frac{1}{n}\right)^{\gamma}}<\frac{1}{\delta^{\gamma}}$. Then

$$
\begin{gathered}
I_{2} \leq \int_{Q_{T} \cap\left\{0 \leq v_{n} \leq \delta\right\} \cap\left\{u_{n}>\delta\right\}} f_{n}(t, x) \frac{\delta}{\delta \gamma} \varphi^{2}(x)=\delta^{1-\gamma} \int_{Q_{T}} f_{n}(t, x) \varphi^{2}(x) \\
\leq \delta^{1-\gamma} \sup _{x \in \Omega}\left|\varphi^{2}(x)\right| \int_{Q_{T}} f_{n}(t, x) \leq C \delta^{1-\gamma}
\end{gathered}
$$

If $\gamma=1$, we consider the decomposition

$$
\begin{gathered}
\int_{Q_{T} \cap\left\{0 \leq v_{n} \leq \delta\right\}} f_{n}(t, x) \frac{v_{n}}{u_{n}+\frac{1}{n}} \varphi^{2}(x) \\
=\int_{Q_{T} \cap\left\{0 \leq v_{n} \leq \delta\right\} \cap\left\{0 \leq u_{n} \leq \sqrt{\delta}\right\}} f_{n}(t, x) \frac{v_{n}}{u_{n}+\frac{1}{n}} \varphi^{2}(x) \\
+\int_{Q_{T} \cap\left\{0 \leq v_{n} \leq \delta\right\} \cap\left\{u_{n}>\sqrt{\delta}\right\}} f_{n}(t, x) \frac{v_{n}}{u_{n}+\frac{1}{n}} \varphi^{2}(x) \\
\leq \int_{Q_{T} \cap\left\{0 \leq u_{n} \leq \sqrt{\delta}\right\}} f_{n}(t, x) \frac{v_{n}}{u_{n}+\frac{1}{v_{n}}} \varphi^{2}(x) \\
+\int_{Q_{T} \cap\left\{0 \leq v_{n} \leq \delta\right\} \cap\left\{u_{n}>\sqrt{\delta}\right\}} f_{n}(t, x) \frac{v_{n}}{u_{n}+\frac{1}{n}} \varphi^{2}(x) \\
=J_{1}+J_{2}
\end{gathered}
$$

Choosing as test function in the equation solved by $u_{n}$ the function

$$
\phi_{\sigma}=\frac{T_{\sigma}\left(-\left(u_{n}-\sqrt{\delta}\right)^{-}\right)}{\sigma} \varphi^{2}(x)
$$

with $\varphi \in C_{0}^{\infty}(\Omega), \varphi \geq 0$, and repeating the same arguments of the proof of (4.6), we obtain

$$
J_{1} \leq C \sqrt{\delta}
$$

For the term $J_{2}$, since $v_{n} \leq \delta$ and $\frac{1}{n}+u_{n}>\sqrt{\delta}$, he comes $\frac{1}{u_{n}+\frac{1}{n}}<\frac{1}{\sqrt{\delta}}$, we obtain

$$
\begin{gathered}
\int_{Q_{T} \cap\left\{0 \leq v_{n} \leq \delta\right\} \cap\left\{u_{n}>\sqrt{\delta}\right\}} f_{n}(t, x) \frac{v_{n}}{u_{n}+\frac{1}{n}} \varphi^{2}(x) \\
\leq \sqrt{\delta} \int_{Q_{T} \cap\left\{0 \leq v_{n} \leq \delta\right\} \cap\left\{u_{n}>\sqrt{\delta}\right\}} f_{n}(t, x) \varphi^{2}(x) \\
\leq \sqrt{\delta} \sup _{x \in \Omega}|\varphi(x)| \int_{Q_{T}} f_{n}(t, x) \leq C \sqrt{\delta}
\end{gathered}
$$

Therefore, by (4.18) - (4.24), we finally obtain (4.7).

We proceed as in the previous cases (i) and (ii), we easily arrive at (4.8) and (4.9). 


\section{Convergence and compactness results}

To pass to the limit as $n \rightarrow \infty$ in (3.4) and (3.5) we need strongly convergent subsequences, which ensured by the following Proposition.

Proposition 5.1. There exists a couple

$$
(u, v) \in\left[L^{2}(0, T ; V) \cap L^{\infty}\left(Q_{T}\right)\right] \times\left[L^{2}(0, T ; W) \cap L^{\infty}\left(Q_{T}\right)\right]
$$

such that, as $n \rightarrow \infty$, we have

$$
\begin{gathered}
u_{n} \rightarrow u \quad \text { weakly in } L^{2}(0, T ; V) \\
v_{n} \rightarrow v \quad \text { weakly in } L^{2}(0, T ; W) \\
u_{n} \rightarrow u \quad \text { weakly in } L^{\infty}\left(Q_{T}\right) \\
v_{n} \rightarrow v \quad \text { weakly in } L^{\infty}\left(Q_{T}\right) \\
u_{n} \rightarrow u \quad \text { strongly in } L^{1}\left(Q_{T}\right) \\
v_{n} \rightarrow v \quad \text { strongly in } L^{1}\left(Q_{T}\right) \\
u_{n} \rightarrow u \quad \text { a.e. in } Q_{T} \\
v_{n} \rightarrow v \quad \text { a.e. in } Q_{T}
\end{gathered}
$$

up to a subsequence.

Proof. Convergences (5.1) and (5.2) are direct consequences of (4.3). The same thing applies to convergences (5.3) and (5.4). To prove (5.5) and (5.7), we observe that the estimate (4.4) leads to

$$
f_{n}(t, x) \frac{v_{n}}{\left(u_{n}+\frac{1}{n}\right)^{\gamma}} \varphi^{2} \in L^{1}\left(Q_{T}\right) \quad, \quad \forall \varphi \in C_{0}^{\infty}(\Omega)
$$

In addition, we have

$$
\frac{\partial\left(u_{n} \varphi^{2}\right)}{\partial t} \text { is bounded in } L^{2}\left(0, T ; V^{*}\right)+L^{1}\left(Q_{T}\right)
$$

By (5.10), choosing $s$ such that $s>\frac{N}{2}+1$, using the same argument as Lemma 2.3 in [25], we deduce that $\frac{\partial\left(u_{n} \varphi\right)}{\partial t}$ is also bounded in $L^{1}\left(0, T ; H^{-s}\right)$. Consequently, since $s>\frac{N}{2}$, we find that

$$
V \subset L^{p}(\Omega) \subset H^{-s}(\Omega)
$$

and the embedding $V \hookrightarrow L^{p}(\Omega)$ is compact. Applying now Corollary 4 in [35], by (5.10) and the compactness results we deduce that $u_{n} \varphi$ is relatively compact in $L^{2}\left(Q_{T}\right)$. Hence, up to a subsequences, convergences (5.5) and (5.7) are satisfied. In the same way for the sequence $\left\{v_{n}\right\}$, we find (5.6) and (5.8).

Proposition 5.2. We have

$$
\begin{aligned}
& \lim _{n \rightarrow \infty} \int_{Q_{T}}\left|\nabla\left(u_{n}-u\right)\right|^{2}=0 \\
& \lim _{n \rightarrow \infty} \int_{Q_{T}}\left|\nabla\left(v_{n}-v\right)\right|^{2}=0
\end{aligned}
$$

Therefore,

$$
\begin{array}{ll}
\nabla u_{n} \rightarrow \nabla u & \text { a.e. in } Q_{T} \\
\nabla v_{n} \rightarrow \nabla v & \text { a.e. in } Q_{T}
\end{array}
$$

Proof. This result can be obtained as a particular case of Proposition 3.14 in [11]. 


\section{Uniform estimate near the singularity}

We consider the set $\left\{(t, x) \in Q_{T}: u(t, x)=0\right.$ a.e. in $\left.Q_{T}\right\}$. As a consequence of the uniform estimate near the singularity (4.6), we have the following Proposition :

Proposition 6.1. The couple $(u, v)$ as a solution to (1.1)-(1.4), in the sense of Definition 2.1, satisfies

$$
\begin{aligned}
& \int_{Q_{T} \cap\{u=0\}} f(t, x) \frac{v}{u^{\gamma}} \psi=0 \\
& \int_{Q_{T} \cap\{u=0\}} g(t, x) \frac{v}{u^{\gamma}} \phi=0
\end{aligned}
$$

for all $\psi, \phi \in C_{0}^{\infty}([0, T) \times \Omega)$ with $\psi, \phi \geq 0$. Moreover, it holds

$$
\begin{aligned}
\int_{Q_{T}} f(t, x) \frac{v}{u^{\gamma}} \psi & =\int_{Q_{T} \cap\{u>0\}} f(t, x) \frac{v}{u^{\gamma}} \psi \\
\int_{Q_{T}} g(t, x) \frac{v}{u^{\gamma}} \phi & =\int_{Q_{T} \cap\{u>0\}} g(t, x) \frac{v}{u^{\gamma}} \phi
\end{aligned}
$$

Proof. (i) We consider a test function $\psi \in C_{0}^{\infty}([0, T) \times \Omega), \psi \geq 0$, with $\operatorname{supp} \psi=$ $\left[0, T_{1}\right] \times Y, T_{1}<T, Y \subset \subset E \subset \subset \Omega$ and $\varphi \in C_{0}^{1}(\Omega)$ with $\varphi(x)=1$ over $Y, \varphi \geq 0$ with $\operatorname{supp} \varphi=E$. By the uniform estimate (4.6), we obtain

$$
\begin{aligned}
& \int_{Q_{T} \cap\left\{u_{n}<\delta\right\}} f_{n}(t, x) \frac{v_{n}}{\left(u_{n}+\frac{1}{n}\right)^{\gamma}} \psi(t, x) \\
& \leq\|\psi\|_{\infty} \int_{[0, T] \times Y} f_{n}(t, x) \frac{v_{n}}{\left(u_{n}+\frac{1}{n}\right)^{\gamma}} \chi_{\left\{u_{n}<\delta\right\}} \\
& \leq\|\psi\|_{\infty} \int_{Q_{T}} f_{n}(t, x) \frac{v_{n}}{\left(u_{n}+\frac{1}{n}\right)^{\gamma}} \varphi^{2}(x) \chi_{\left\{u_{n}<\delta\right\}} \leq C \delta
\end{aligned}
$$

On the other hand,

$$
\begin{gathered}
\int_{Q_{T}} f_{n}(t, x) \frac{v_{n}}{\left(u_{n}+\frac{1}{n}\right)^{\gamma}} \chi_{\left\{u_{n}<\delta\right\}} \psi(t, x) \\
=\int_{Q_{T}} f_{n}(t, x) \frac{v_{n}}{\left(u_{n}+\frac{1}{n}\right)^{\gamma}} \chi_{\left\{u_{n}<\delta\right\}} \chi_{\{u=\delta\}} \psi(t, x) \\
+\int_{Q_{T}} f_{n}(t, x) \frac{v_{n}}{\left(u_{n}+\frac{1}{n}\right)^{\gamma}} \chi_{\left\{u_{n}<\delta\right\}} \chi_{\{u \neq \delta\}} \psi(t, x) \leq C \delta
\end{gathered}
$$

We observe that there exists at most a countable set $D_{1}$ such that meas $\{(t, x): u(t, x)=$ $\delta\}>0$. We choose $\delta$ outside of this set $D_{1}$, so that, in (6.5), the integral

$$
\int_{Q_{T}} f_{n}(t, x) \frac{v_{n}}{\left(u_{n}+\frac{1}{n}\right)^{\gamma}} \chi_{\left\{u_{n}<\delta\right\}} \chi_{\{u=\delta\}} \psi(t, x)=0
$$

So, we have

$$
\begin{gathered}
\int_{Q_{T}} f_{n}(t, x) \frac{v_{n}}{\left(u_{n}+\frac{1}{n}\right)^{\gamma}} \chi_{\left\{u_{n}<\delta\right\}} \psi(t, x) \\
=\int_{Q_{T}} f_{n}(t, x) \frac{v_{n}}{\left(u_{n}+\frac{1}{n}\right)^{\gamma}} \chi_{\left\{u_{n}<\delta\right\}} \chi_{\{u \neq \delta\}} \psi(t, x) \leq C \delta
\end{gathered}
$$

Since by (5.7),

$$
\chi_{\left\{u_{n}<\delta\right\}} \chi_{\{u \neq \delta\}} \rightarrow \chi_{\{u<\delta\}} \text { a.e. in } Q_{T}
$$

Applying Fatou's Lemma in (6.6) for $\delta$ fixed, leads to

$$
\int_{Q_{T}} f(t, x) \frac{v}{u^{\gamma}} \chi_{\{u<\delta\}} \psi(t, x) \leq C \delta
$$


Using again Fatou's Lemma in the last inequality for $\delta \rightarrow 0$, we get

$$
\int_{Q_{T}} f(t, x) \frac{v}{u^{\gamma}} \chi_{\{u=0\}} \psi(t, x)=\int_{Q_{T} \cap\{u=0\}} f(t, x) \frac{v}{u^{\gamma}} \psi(t, x)=0
$$

this leads to

$$
\int_{Q_{T}} f(t, x) \frac{v}{u^{\gamma}} \psi(t, x)=\int_{Q_{T} \cap\{u>0\}} f(t, x) \frac{v}{u^{\gamma}} \psi(t, x)
$$

which are the desired identities.

(ii) In the same way with some simplifications, we prove (6.2) and (6.4). We consider a test function $\phi \in C_{0}^{\infty}([0, T) \times \Omega), \phi \geq 0$, with supp $\phi=\left[0, T_{2}\right] \times Y, T_{2}<T, \bar{Y} \subset \subset E \subset \subset \Omega$ and $\varphi \in C_{0}^{1}(\Omega)$ with $\varphi(x)=1$ over $\bar{Y}, \varphi \geq 0$ with $\operatorname{supp} \varphi=E$. By the uniform estimate (4.9), we obtain

$$
\begin{aligned}
& \int_{Q_{T} \cap\left\{u_{n}<\delta\right\}}\left|g_{n}(t, x)\right| \frac{v_{n}}{\left(u_{n}+\frac{1}{n}\right)^{\gamma}} \phi(t, x) \\
& \leq\|\phi\|_{\infty} \int_{[0, T] \times Y}\left|g_{n}(t, x)\right| \frac{v_{n}}{\left(u_{n}+\frac{1}{n}\right)^{\gamma}} \chi_{\left\{u_{n}<\delta\right\}} \\
& \leq\|\phi\|_{\infty} \int_{Q_{T}}\left|g_{n}(t, x)\right| \frac{v_{n}}{\left(u_{n}+\frac{1}{n}\right)^{\gamma}} \varphi^{2}(x) \chi_{\left\{u_{n}<\delta\right\}} \\
& \leq \begin{cases}C \delta^{1-\gamma} & \text { if } 0<\gamma<1 \\
C \sqrt{\delta} & \text { if } \gamma=1\end{cases}
\end{aligned}
$$

On the other hand,

$$
\begin{gathered}
\int_{Q_{T}}\left|g_{n}(t, x)\right| \frac{v_{n}}{\left(u_{n}+\frac{1}{n}\right)^{\gamma}} \chi_{\left\{u_{n}<\delta\right\}} \phi(t, x) \\
=\int_{Q_{T}}\left|g_{n}(t, x)\right| \frac{v_{n}}{\left(u_{n}+\frac{1}{n}\right)^{\gamma}} \chi_{\left\{u_{n}<\delta\right\}} \chi_{\{u=\delta\}} \phi(t, x) \\
+\int_{Q_{T}}\left|g_{n}(t, x)\right| \frac{v_{n}}{\left(u_{n}+\frac{1}{n}\right)^{\gamma}} \chi_{\left\{u_{n}<\delta\right\}} \chi_{\{u \neq \delta\}} \phi(t, x) \\
\leq \begin{cases}C \delta^{1-\gamma} & \text { if } 0<\gamma<1 \\
C \sqrt{\delta} & \text { if } \gamma=1\end{cases}
\end{gathered}
$$

We observe that there exists at most a countable set $\bar{D}_{2}$ such that meas $\{(t, x): u(t, x)=$ $\delta\}>0$. We choose $\delta$ outside of this set $\bar{D}_{2}$, so that, in (6.9), the integral

$$
\int_{Q_{T}}\left|g_{n}(t, x)\right| \frac{v_{n}}{\left(u_{n}+\frac{1}{n}\right)^{\gamma}} \chi_{\left\{u_{n}<\delta\right\}} \chi_{\{u=\delta\}} \phi(t, x)=0
$$

So, we have

$$
\begin{gathered}
\int_{Q_{T}}\left|g_{n}(t, x)\right| \frac{v_{n}}{\left(u_{n}+\frac{1}{n}\right)^{\gamma}} \chi_{\left\{u_{n}<\delta\right\}} \phi(t, x) \\
=\int_{Q_{T}}\left|g_{n}(t, x)\right| \frac{v_{n}}{\left(u_{n}+\frac{1}{n}\right)^{\gamma}} \chi_{\left\{u_{n}<\delta\right\}} \chi_{\{u \neq \delta\}} \phi(t, x) \\
\leq \begin{cases}C \delta^{1-\gamma} & \text { if } 0<\gamma<1 \\
C \sqrt{\delta} & \text { if } \gamma=1\end{cases}
\end{gathered}
$$

Since by (5.7),

$$
\chi_{\left\{u_{n}<\delta\right\}} \chi_{\{u \neq \delta\}} \rightarrow \chi_{\{u<\delta\}} \text { a.e. in } Q_{T}
$$

Applying Fatou's Lemma in (6.10) for $\delta$ fixed, leads to

$$
\int_{Q_{T}}\left|g_{n}(t, x)\right| \frac{v}{u^{\gamma}} \chi_{\{u<\delta\}} \phi(t, x) \leq \begin{cases}C \delta^{1-\gamma} & \text { if } 0<\gamma<1 \\ C \sqrt{\delta} & \text { if } \gamma=1\end{cases}
$$


Using again Fatou's Lemma in the last inequality for $\delta \rightarrow 0$, we get

$$
\int_{Q_{T}}|g(t, x)| \frac{v}{u^{\gamma}} \chi_{\{u=0\}} \phi(t, x)=\int_{Q_{T} \cap\{u=0\}}|g(t, x)| \frac{v}{u^{\gamma}} \phi(t, x)=0
$$

this leads to

$$
\int_{Q_{T}}|g(t, x)| \frac{v}{u^{\gamma}} \phi(t, x)=\int_{Q_{T} \cap\{u>0\}}|g(t, x)| \frac{v}{u^{\gamma}} \phi(t, x)
$$

which also means that

$$
\int_{Q_{T}} g(t, x) \frac{v}{u^{\gamma}} \phi(t, x)=\int_{Q_{T} \cap\{u>0\}} g(t, x) \frac{v}{u^{\gamma}} \phi(t, x)
$$

this is the desired result

\section{Proof of the main result}

Now, we give the proof of the main result of this paper. Since $u_{n}, v_{n} \geq 0$ a.e. in $Q_{T}$, thanks to (5.3) and (5.4) we obtain $u, v \geq 0$. Thanks to the convergences (5.5) and (5.6), we can now go to the limit in the parts involving the time derivatives of (3.4) and (3.5).

By (5.11) and (5.12), the sequences $\left(\nabla u_{n}\right)$ and $\left(\nabla v_{n}\right)$ are equi-integrable. By (5.7), (5.8), (5.13) and (5.14), thanks to Vitali's Theorem (see Theorem 1.0.16 in [19]), we obtain

$$
\begin{aligned}
& \nabla u_{n} \rightarrow \nabla u \quad \text { in } L^{2}\left(Q_{T}\right) \\
& \nabla v_{n} \rightarrow \nabla v \quad \text { in } L^{2}\left(Q_{T}\right)
\end{aligned}
$$

We deal now with the singular lower order terms. Let be $D=\left[0, T_{1}\right] \times K, T_{1}<T$, such that $K \subset \subset E \subset \subset \Omega$ and $\psi \in C_{0}^{\infty}([0, T) \times \Omega)$ with $\operatorname{supp} \psi=D$. Let $\varphi$ be a function such that $\varphi(x)=1$ on the set $K, 0 \leq \varphi \leq 1$ and $\operatorname{supp}(\varphi)=E$. For any $\delta>0$ we have

$$
\begin{gathered}
\int_{Q_{T}} f_{n}(t, x) \frac{v_{n}}{\left(u_{n}+\frac{1}{n}\right)^{\gamma}} \psi(t, x) \\
=\int_{Q_{T} \cap\left\{0 \leq u_{n}<\delta\right\}} f_{n}(t, x) \frac{v_{n}}{\left(u_{n}+\frac{1}{n}\right) \gamma} \psi(t, x) \\
+\int_{Q_{T} \cap\left\{u_{n} \geq \delta\right\}} f_{n}(t, x) \frac{v_{n}}{\left(u_{n}+\frac{1}{n}\right)^{\gamma}} \psi(t, x)
\end{gathered}
$$

Concerning the term $A$, we proceed as follows :

$$
\begin{aligned}
A & \leq\|\psi\|_{\infty} \int_{D \cap\left\{0 \leq u_{n}<\delta\right\}} f_{n}(t, x) \frac{v_{n}}{\left(u_{n}+\frac{1}{n}\right)^{\gamma}} \varphi^{2}(x) \\
& \leq\|\psi\|_{\infty} \int_{Q_{T} \cap\left\{0 \leq u_{n}<\delta\right\}} f_{n}(t, x) \frac{v_{n}}{\left(u_{n}+\frac{1}{n}\right)^{\gamma}} \varphi^{2}(x)
\end{aligned}
$$

By (4.6), we get to

$$
A \leq C \delta
$$

where $C$ is a constant independent of $n$. For the term $B$, we see that

$$
\begin{aligned}
B & =\int_{Q_{T}} f_{n}(t, x) \frac{v_{n}}{\left(u_{n}+\frac{1}{n}\right)^{\gamma}} \chi_{\left\{u_{n} \geq \delta\right\}} \chi_{\{u \neq \delta\}} \psi(t, x) \\
& +\int_{Q_{T}} f_{n}(t, x) \frac{v_{n}}{\left(u_{n}+\frac{1}{n}\right)^{\gamma}} \chi_{\left\{u_{n} \geq \delta\right\}} \chi_{\{u=\delta\}} \psi(t, x) \\
& =B_{1}+B_{2}
\end{aligned}
$$


We observe that there is at most a countable set $\mathcal{O}_{1}$ such that meas $\{(t, x): u(t, x)=$ $\delta\}>0$. We choose $\delta$ outside of this set $\mathcal{O}_{1}$, so that the term $B_{2}$ is zero. Since (5.7) holds, for the term $B_{1}$ we have that

$$
\begin{gathered}
\chi_{\left\{u_{n} \geq \delta\right\}} \chi_{\{u \neq \delta\}} \rightarrow \chi_{\{u>\delta\}} \quad \text { a.e. in } Q_{T} \\
f_{n}(t, x) \frac{v_{n}}{\left(u_{n}+\frac{1}{n}\right)^{\gamma}} \chi_{\left\{u_{n} \geq \delta\right\}} \chi_{\{u \neq \delta\}} \psi(x, t) \leq f(t, x) \frac{v_{n}}{\delta^{\gamma}} \psi(t, x) \in L^{1}\left(Q_{T}\right)
\end{gathered}
$$

Thanks to (5.7) and (5.8), the Lebesgue Dominate Convergence Theorem guarantees that

$$
\begin{aligned}
& \lim _{n \rightarrow+\infty} \int_{Q_{T}} f_{n}(t, x) \frac{v_{n}}{\left(u_{n}+\frac{1}{n}\right)^{\gamma}} \chi_{\left\{u_{n} \geq \delta\right\}} \chi_{\{u \neq \delta\}} \psi(t, x) \\
= & \int_{Q_{T}} f(t, x) \frac{v}{u^{\gamma}} \chi_{\{u>\delta\}} \psi(t, x)
\end{aligned}
$$

Then

$$
\lim _{n \rightarrow+\infty} B=\int_{Q_{T}} f(t, x) \frac{v}{u^{\gamma}} \chi_{\{u>\delta\}} \psi(t, x)
$$

By (7.3), (7.4), (7.5) and (6.8), we can deduce that

$$
\begin{aligned}
\lim _{n \rightarrow \infty} \int_{Q_{T}} f_{n}(t, x) \frac{v_{n}}{\left(u_{n}+\frac{1}{n}\right)^{\gamma}} \psi(t, x) & =\lim _{\substack{\delta \rightarrow 0 \\
n \rightarrow \infty}} \int_{Q_{T}} f_{n}(t, x) \frac{v_{n}}{\left(u_{n}+\frac{1}{n}\right)^{\gamma}} \psi(t, x) \chi_{\left\{u_{n}>\delta\right\}} \\
& =\int_{Q_{T} \cap\{u>0\}} f(t, x) \frac{v}{u^{\gamma}} \psi(t, x) \\
& =\int_{Q_{T}} f(t, x) \frac{v}{u^{\gamma}} \psi(t, x)
\end{aligned}
$$

In the same way, we have for any $\delta>0$

$$
\begin{gathered}
\int_{Q_{T}} g_{n}(t, x) \frac{v_{n}}{\left(u_{n}+\frac{1}{n}\right)^{\gamma}} \psi(t, x) \\
=\int_{Q_{T} \cap\left\{0 \leq u_{n}<\delta\right\}} g_{n}(t, x) \frac{v_{n}}{\left(u_{n}+\frac{1}{n}\right)^{\gamma}} \psi(t, x) \\
+\int_{Q_{T} \cap\left\{u_{n} \geq \delta\right\}} g_{n}(t, x) \frac{v_{n}}{\left(u_{n}+\frac{1}{n}\right)^{\gamma}} \psi(t, x) \\
=\bar{A}+\bar{B}
\end{gathered}
$$

Concerning the term $\bar{A}$, we have

$$
|\bar{A}| \leq\|\psi\|_{\infty} \int_{Q_{T} \cap\left\{0 \leq u_{n}<\delta\right\}}\left|g_{n}(t, x)\right| \frac{v_{n}}{\left(u_{n}+\frac{1}{n}\right)^{\gamma}} \varphi^{2}(x)
$$

By (4.9), we get to

$$
|\bar{A}| \leq\|\psi\|_{\infty} \cdot \begin{cases}C \delta^{1-\gamma} & \text { if } 0<\gamma<1 \\ C \sqrt{\delta} & \text { if } \gamma=1\end{cases}
$$

which implies $\lim _{\delta \rightarrow 0}|\bar{A}|=0$, where $C$ is a constant independent of $n$. For the term $\bar{B}$, we see that

$$
\begin{aligned}
\bar{B} & =\int_{Q_{T}} g_{n}(t, x) \frac{v_{n}}{\left(u_{n}+\frac{1}{n}\right)^{\gamma}} \chi_{\left\{u_{n} \geq \delta\right\}} \chi_{\{u \neq \delta\}} \psi(t, x) \\
& +\int_{Q_{T}} g_{n}(t, x) \frac{v_{n}}{\left(u_{n}+\frac{1}{n}\right)^{\gamma}} \chi_{\left\{u_{n} \geq \delta\right\}} \chi_{\{u=\delta\}} \psi(t, x) \\
& =\bar{B}_{1}+\bar{B}_{2}
\end{aligned}
$$


We observe that there is at most a countable set $\mathcal{O}_{2}$ such that meas $\{(t, x): u(t, x)=$ $\delta\}>0$. We choose $\delta$ outside of this set $\mathcal{O}_{2}$, so that the term $\bar{B}_{2}$ is zero. Since (5.7) holds, for the term $\bar{B}_{1}$ we have that

$$
\begin{gathered}
\chi_{\left\{u_{n} \geq \delta\right\}} \chi_{\{u \neq \delta\}} \rightarrow \chi_{\{u>\delta\}} \quad \text { a.e. in } Q_{T} \\
\left|g_{n}(t, x)\right| \frac{v_{n}}{\left(u_{n}+\frac{1}{n}\right) \gamma} \chi_{\left\{u_{n} \geq \delta\right\}} \chi_{\{u \neq \delta\}} \psi(x, t) \leq|g(t, x)| \frac{v_{n}}{\delta \gamma} \psi(t, x) \in L^{1}\left(Q_{T}\right)
\end{gathered}
$$

Thanks to (5.7) and (5.8), the Lebesgue Dominate Convergence Theorem guarantees that

$$
\lim _{n \rightarrow \infty} \bar{B}=\int_{Q_{T}} g(t, x) \frac{v}{u^{\gamma}} \chi_{\{u>\delta\}} \psi(t, x)
$$

By (7.6), (7.7), (7.8) and (6.8), we can deduce

$$
\begin{aligned}
\lim _{n \rightarrow \infty} \int_{Q_{T}} g_{n}(t, x) \frac{v_{n}}{\left(u_{n}+\frac{1}{n}\right)^{\gamma}} \psi(t, x) & =\lim _{\substack{\delta \rightarrow 0 \\
n \rightarrow \infty}} \int_{Q_{T}} g_{n}(t, x) \frac{v_{n}}{\left(u_{n}+\frac{1}{n}\right)^{\gamma}} \psi(t, x) \chi_{\left\{u_{n}>\delta\right\}} \\
& =\int_{Q_{T} \cap\{u>0\}} g(t, x) \frac{v}{u^{\gamma}} \psi(t, x) \\
& =\int_{Q_{T}} g(t, x) \frac{v}{u^{\gamma}} \psi(t, x)
\end{aligned}
$$

Repeating the same argument for $u_{n}$ to deal with the case of $v_{n}$, but this time we use (4.7) and (4.8), which ends the proof of our main result Theorem 2.2.

\section{Concluding remarks and perspectives}

This work has mainly focused on the question of the existence of weak solutions for a class of singular reaction diffusion systems. Many important results have been obtained with additional assumptions that can be applied to extinction models and other models in biology, ecology, physics, and others as appropriate.

We have developed original methods to overcome certain difficulties, and despite the complexity of the model studied, we have succeeded in obtaining an existence result.

In addition to this work, we can address the following interesting questions :

- Question of uniqueness, by considering the notion of entropic solutions.

- Mathematical analysis of anisotropic system, which consists in adding diffusion coefficients to the studied system depending on $(t, x)$ or more generally depending on $(t, x, u, \nabla u)$.

- Asymptotic behaviour of solutions.

- Numerical simulation.

This list of loose themes corresponds to work in progress or prospective. Some are a continuation of the work already done, and some are new research projects.

This not only makes it possible to be closer to the reality and concerns of the current industrial world, but also goes beyond the theoretical framework by developing models and tools that can be used and transferred to various industries.

\section{References}

[1] N. Alaa, S. Mesbahi and W. Bouarifi, Global existence of weak solutions for parabolic triangular reaction diffusion systems applied to a climate model, An. Univ. Craiova Ser. Mat. Inform. 42 (1), 80-97, 2015.

[2] N. Alaa, S. Mesbahi, A. Mouida and W. Bouarifi, Existence of solutions for quasilinear elliptic degenerate systems with $L^{1}$ data and nonlinearity in the gradient. Electron. J. Differential Equations 2013 (142), 1-13, 2013. 
[3] R. Aris, Mathematical Modeling : A Chemical Engineer's Perspective, Academic Press, New York, 1999.

[4] M.A. Beauregard and Q. Sheng, A fully adaptive approximation for quenching-type reaction diffusion equations over circular domains, Numerical methods in PDE, 30 (2), 472-489, 2013.

[5] L. Boccardo and L. Orsina, Semilinear elliptic equations with singular nonlinearities, Calc. Var. Partial Differential Equations, 37, 363-380, 2009.

[6] P. Constantin, A. Kiselev and L. Ryzhik, Quenching of flames by fluid advection, Comm. Pure Appl. Math. 54, 1320-1342, 2001.

[7] Q. Dai and Y. Gu, A Short Note on Quenching Phenomena for Semilinear Parabolic Equations, J. Differential Equations, 137, 240-250, 1997.

[8] A. Dall'Aglio, D. Giachetti and J.P. Puel, Nonlinear parabolic equations with natural growth in general domains, Boll. Unione Mat. Ital. Serie 8 8-B (3), 653-683, 2005.

[9] I. de Bonis and A. Muntean, Existence of weak solutions to a nonlinear reaction diffusion system with singular sources, Electron. J. Differential Equations 2017 (202), $1-16,2017$.

[10] I. de Bonis, Singular elliptic and parabolic problems : existence and regularity of solutions, Ph D Thesis, Sapienza University of Rome, 2015.

[11] I. de Bonis and D. Giachetti, Nonnegative solutions for a class of singular parabolic problems involving p-laplacian, Asymptot. Anal. 91, 147-183, 2015.

[12] I. de Bonis and L.M. De Cave, Degenerate parabolic equations with singular lower order terms, Differential Integral Equations, 27 (9/10), 949-976, 2014.

[13] I. de Bonis and D. Giachetti, Singular parabolic problems with possibly changing sign data, Discrete Contin. Dyn. Syst. Ser. B 7 (19), 2047-2064, 2014.

[14] D. Giachetti, Martinez-Aparicio and P.J., Murat, A semilinear elliptic equation with a mild singularity at $u=0$ : existence and homogenization, J. Math. Pures Appl. 2016.

[15] H. Kawarada, On solutions of the initial boundary value problem for $u_{t}=u_{x x}+1$, RIMS Kyoto 10, 729-736, 1975.

[16] A. Kiselev and A. Zlatos, Quenching of combustion by shear flows, Duke Math. J. 132, 49-72, 2006.

[17] R. Landes and V. Mustonen, On parabolic initial-boundary value problems with critical growth for the gradient, Ann. Inst. H. Poincaré Anal. Non Linéaire, 11, 135-158, 1994.

[18] R. Landes, On the existence of weak solutions for quasilinear parabolic initial-boundary value problems, Proc. Roy. Soc. Edinburgh Sect. A 89, 217-237, 1981.

[19] O. Levenspiel, Chemical Reaction Engineering, John Wiley and Sons, New York, 1962.

[20] H.A. Levine, The phenomenon of quenching : A survey, Proceedings, VIth International Conference on Trends in the Theory and Practice of Nonlinear Analysis, North-Holland, New York, 1985.

[21] J.L. Lions, Quelques méthodes de résolution des problémes aux limites non linéaires, Dunod et Gautier-Villars, Paris, 1969.

[22] P.T. Marion, Quenching Phenomena Due to a Concentrated Nonlinear Source in an Infinitely Long Cylinder, Journal of Applied Mathematics and Physics 7, 2015-2025, 2019.

[23] S. Mesbahi and N. Alaa, Mathematical analysis of a reaction diffusion model for image restoration, An. Univ. Craiova Ser. Mat. Inform. 42 (1), 70-79, 2015.

[24] S. Mesbahi and N. Alaa, Existence result for triangular Reaction Diffusion systems with $L^{1}$ data and critical growth with respect to the gradient, Mediterr. J. Math. 10, 255-275, 2013.

[25] A. Mokrane, Existence of bounded solutions of some nonlinear parabolic equations, Proc. Roy. Soc. Edinburgh Sect. A 107, 313-326, 1987. 
[26] A. Muntean, Continuum Modeling: An Approach through Practical Examples, Springer Briefs in Applied Science and Technology : Mathematical Methods, Springer, Heidelberg, 2015.

[27] J.D. Murray, Mathematical Biology I : An Introduction, vol. I, 3rd ed., SpringerVerlag, Berlin Heidelberg, 2003.

[28] J.D. Murray, Mathematical Biology II : Spatial Models and Biochemical Applications, vol. II, 3rd ed., Springer-Verlag, Berlin Heidelberg, 2003.

[29] M. Pierre, Global existence in reaction diffusion systems with control of mass: a survey, Milan J. Math. 78, 417-455, 2010.

[30] T. Salin, Quenching and blowup problems for reaction diffusion equations, Helsinki University of Technology Institute of Mathematics Research Reports A466, 2004.

[31] T. Salin, On quenching with logarithmic singularity, Nonlinear Anal. TMA. 52, 261$289,2003$.

[32] T. Salin, Quenching-rate estimate for a reaction diffusion equation with weakly singular reaction term, Dyn. Contin. Discrete Impuls. Syst. Ser. A Math. Anal. 11 (4), 2004.

[33] T. Salin, On a refined asymptotic analysis for the quenching problem, Helsinki University of Technology, Institute of Mathematics, Research Report A457, 2003.

[34] B. Selçuk, Quenching behavior of a semilinear reaction diffusion system with singular boundary condition, Turk J Math. 40, 166-180, 2016.

[35] J. Simon, Compact sets in the space $L^{p}(0, T, B)$, Ann. Mat. Pura Appl. 146, 65-96, 1987.

[36] G. Stampacchia, Le problème de Dirichlet pour les équations elliptiques du second ordre à coefficients discontinus, Ann. Inst. Fourier (Grenoble,) 15, 189-258, 1965.

[37] Q. Wang, Quenching Phenomenon for a Parabolic MEMS Equation, Chin. Ann. Math. Ser. B, 39 (1), 129-144, 2018. 